\title{
Involvement of hypoxia-triggered endoplasmic reticulum stress in outlet obstruction-induced apoptosis in the urinary bladder
}

\author{
Norifumi Sawada ${ }^{1,2}$, Jian Yao ${ }^{1}$, Nobuhiko Hiramatsu, Kunihiro Hayakawa ${ }^{1}$, Isao Araki ${ }^{2}$, Masayuki Takeda ${ }^{2}$ and \\ Masanori Kitamura ${ }^{1}$
}

In bladder outlet obstruction (BOO), mechanical stress and ischemia/hypoxia are implicated in structural and functional alterations of the urinary bladder. Because mechanical stress and hypoxia may trigger endoplasmic reticulum (ER) stress, we examined involvement of ER stress in the damage of the bladder caused by BOO. An experimental model of $\mathrm{BOO}$ was established in rats by complete ligature of the urethra for $24 \mathrm{~h}$, and bladders were subjected to northern blot analysis and assessment of apoptosis. Isolated urinary bladders and bladder-derived smooth muscle cells (BSMCs) were also exposed to mechanical strain and hypoxia and used for analyses. To examine involvement of ER stress in the damage of the bladder, the effects of a chemical chaperone 4-phenylbutyrate (4-PBA) were evaluated in vitro and in vivo. Outlet obstruction for $24 \mathrm{~h}$ induced expression of ER stress markers, GRP78 and CCAAT/enhancer-binding protein-homologous protein $(C H O P)$, in the bladder. It was associated with induction of markers for mechanical stress (cyclooxygenases 2) and hypoxia (vascular endothelial growth factor and glyceraldehyde-3-phosphate dehydrogenase). When isolated bladders and BSMCs were subjected to mechanical strain, induction of GRP78 and CHOP was not observed. In contrast, when BSMCs were exposed to hypoxic stress caused by $\mathrm{CoCl}_{2}$ or thenoyltrifluoroacetone (TTFA), substantial upregulation of GRP78 and CHOP was observed, suggesting involvement of hypoxia in the induction of ER stress. In the bladder subjected to $\mathrm{BOO}$, the number of terminal deoxynucleotidyl transferase-mediated dUTP nick-end labeling-positive cells increased in the epithelial cells and BSMCs. Similarly, treatment with TTFA or $\mathrm{CoCl}_{2}$ induced apoptosis of BSMCs, and 4-PBA significantly attenuated ER stress and apoptosis triggered by these agents. Furthermore, in vivo administration with 4-PBA significantly reduced apoptosis in the bladder subjected to $\mathrm{BOO}$. These results suggested that outlet obstruction caused ER stress via hypoxic stress in the bladder and that hypoxia-triggered ER stress may be involved in the induction of apoptosis in BOO.

Laboratory Investigation (2008) 88, 553-563; doi:10.1038/labinvest.2008.21; published online 17 March 2008

KEYWORDS: bladder outlet obstruction; hypoxia; endoplasmic reticulum stress; bladder smooth muscle cell; apoptosis

Bladder outlet obstruction (BOO) is a common outcome of benign prostatic hypertrophy, urethral stricture and congenital anomalies of the posterior urethral valve. Regardless of these initial causes of BOO, compensatory responses occur in the bladder, leading to its dysfunction and structural alterations including reduced bladder elasticity and micturitional problems. ${ }^{1-3}$ Histopathologically, bladders respond to the obstructive stress by cell proliferation, cellular hypertrophy and accumulation of extracellular matrix. If the stress is beyond the capacity of the compensatory responses, the cells undergo apoptosis. Although multiple triggers and mediators have been postulated to contribute to the remodeling of the bladder, ${ }^{1}$ the signaling mechanisms involved have not been fully elucidated. Several previous reports suggested that excessive distension of the bladder caused ischemia and consequent hypoxia, which may be one of the major triggers to induce bladder dysfunction in BOO., Hypoxia may also trigger the proapoptotic machinery in the bladder, leading to cellular and tissue damage. Another line of evidence suggested that mechanical stress on the bladder

\footnotetext{
'Department of Molecular Signaling, Interdisciplinary Graduate School of Medicine and Engineering, University of Yamanashi, Chuo, Yamanashi, Japan and 2Department of Urology, Interdisciplinary Graduate School of Medicine and Engineering, University of Yamanashi, Chuo, Yamanashi, Japan

Correspondence: Professor M Kitamura, MD, PhD, Department of Molecular Signaling, Interdisciplinary Graduate School of Medicine and Engineering, University of Yamanashi, Shimokato 1110, Chuo, Yamanashi 409-3898, Japan.

E-mail: masanori@yamanashi.ac.jp

Received 29 October 2007; revised 14 February 2008; accepted 14 February 2008
} 
wall may cause bladder hypertrophy and detrusor overactivity. The repetitive stretch of bladder smooth muscle cells (BSMCs) resulted in their structural and functional alterations via the activation of stretch-activated ion channels and several kinases, including protein kinase $\mathrm{C}$ and $\mathrm{c}$-Jun $\mathrm{N}$-terminal kinase (JNK), leading to the expression of an array of genes including cyclooxygenases 2 (COX-2). ${ }^{4}$ However, molecular mechanisms underlying BOO-associated cellular dysfunction are still largely unknown.

Previous reports suggested that in other organs, mechanical stress and hypoxia trigger a particular stress response, the endoplasmic reticulum (ER) stress response. ER stress is defined as the accumulation of unfolded or misfolded proteins in the ER, which induces a coordinated adaptive program called unfolded protein response (UPR). UPR alleviates ER stress by suppression of protein synthesis and facilitation of protein folding via induction of ER chaperones, including $78 \mathrm{kDa}$ glucose-regulated protein (GRP78) and reinforced degradation of unfolded proteins. If the stress is beyond the capacity of the adaptive machinery, however, the cells undergo apoptosis via several mechanisms, including the induction of CCAAT/enhancer-binding protein-homologous protein (CHOP). ${ }^{5}$ A recent report showed that ER stress was involved in the induction of apoptosis of cardiomyocytes during cardiac pressure overload caused by aortic constriction. ${ }^{6}$ Other recent reports also indicated involvement of ER stress in the ischemic/hypoxic insults in the brain and heart. $^{7,8}$

In the present investigation, we aimed at testing our hypothesis that under the outlet obstruction, ER stress may be induced in the bladder via mechanical stress or hypoxia and thereby contribute to apoptotic tissue injury. Using cultured BSMCs, isolated bladders and an in vivo model of $\mathrm{BOO}$, we here demonstrate that (1) BOO causes ER stress in the bladder, (2) the induction of ER stress is not via mechanical stress but via hypoxic stress and (3) hypoxia-triggered ER stress may be involved in the induction of apoptosis in the bladder suffered from BOO.

\section{MATERIALS AND METHODS \\ Reagents}

$\mathrm{CoCl}_{2}$ and sodium 4-phenylbutyrate (4-PBA) were purchased from Wako Pure Chemical Industries (Osaka, Japan). 2-Thenoyltrifluoroacetone (TTFA), Hoechst33258, 4',6-diamidino-2-phenylindole (DAPI), tunicamycin and thapsigargin were obtained from Sigma-Aldrich Japan (Tokyo, Japan).

\section{Establishment of BSMCs}

BSMCs were established from the bladder of female SpragueDawley rats as described previously. ${ }^{9}$ The bladder body was placed on ice, and the outer layers (tunica serosa and tunica adventitia) and the inner layers (tunica intima and the epithelium) were removed. The remaining tunica media was also removed with a cotton swab. The smooth muscle layer was then incubated with shaking at $37^{\circ} \mathrm{C}$ for $30 \mathrm{~min}$ in phosphate-buffered saline containing $0.2 \%$ trypsin. After the incubation, the tissue was minced and suspended in Dulbecco's modified Eagle's medium nutrient mixture F-12 (DMEM/ F-12; GIBCO-BRL, Gaithersburg, MD, USA) supplemented with $0.1 \%$ collagenase using Pasteur pipettes. The suspension was further incubated at $37^{\circ} \mathrm{C}$ for $30 \mathrm{~min}$ and centrifuged for $5 \mathrm{~min}$. The pellet was resuspended in DMEM/F-12 containing $10 \%$ fetal bovine serum (FBS) and centrifuged at $250 \mathrm{G}$ for $2 \mathrm{~min}$, and the supernatant fraction containing BSMCs was used for culture. The SMC-phenotype of the established cells was confirmed by positive staining for $\alpha$-actin. ${ }^{10}$ The cells between the 11-20th passages were used for experiments.

\section{Experimental Model of BOO}

Female Sprague-Dawley rats (190-240 g) were anesthetized with sodium pentobarbital $(10 \mathrm{mg} / \mathrm{kg})$, and $\mathrm{BOO}$ was established by a tight ligature of the proximal urethra using a 4-0 silk thread. After $24 \mathrm{~h}$, bladders were isolated and subjected to northern blot analysis and histological analysis. For the latter, frozen sections were prepared and used for immunohistochemistry, hematoxylin-eosin staining and terminal deoxynucleotidyl transferase-mediated dUTP nick-end labeling (TUNEL), as described later. Sham operation was performed without ligature of the urethra. To examine the effects of 4-PBA, rats were administered daily with either ethanol or 4-PBA $(120 \mathrm{mg} / \mathrm{kg}$, i.p.) for 3 days prior to BOO. Experiments were conducted with the approval of the committee for animal experiments at the University of Yamanashi. Animals were treated humanely and with regard for alleviation of suffering.

\section{Ex Vivo Model of Mechanical Stress on the Bladder}

RPMI1640 medium (3 ml; GIBCO-BRL) was injected into the bladder in vivo through a $22 \mathrm{~F}$ angiocatheter (B Braun Melsungen AG, PA, USA) cannulated into the urethra in anesthetized female rats. After the injection, the urethra was tied up tightly with a ligature, and the distended bladder was isolated. The isolated bladder was incubated at $37^{\circ} \mathrm{C}$ for $6 \mathrm{~h}$ in an organ bath apparatus filled with Krebs-Henseleit solution $\left(\mathrm{NaCl} 113 \mathrm{mEq}, \mathrm{KCl} 4.7 \mathrm{mEq}, \mathrm{CaCl}_{2} 2.5 \mathrm{mEq}, \mathrm{MgSO}_{4}\right.$ $1.2 \mathrm{mEq} \mathrm{NaHCO}_{3} 25 \mathrm{mEq}, \mathrm{KH}_{2} \mathrm{PO}_{4} 1.2 \mathrm{mEq}$ and glucose $11.5 \mathrm{mEq}$ ) supplied with $95 \% \mathrm{O}_{2}$ and $5 \% \mathrm{CO}_{2}$ and subjected to northern blot analysis, as described later.

\section{In Vitro Model of Mechanical Stress on BSMCs}

BSMCs suspended in DMEM/F-12 containing 1\% FBS were seeded on deformable silicone chambers precoated with type I collagen. After achieving 90\% confluency, cells were subjected to static mechanical stretch by $40 \%$ for up to $24 \mathrm{~h}$ using special metal frames, ${ }^{11}$ as described previously.

\section{Northern Blot Analysis}

Total RNA was extracted by a single-step method, and northern blot analysis was performed as described before. ${ }^{12}$ 
cDNAs for GRP78, ${ }^{13} \mathrm{CHOP},{ }^{14} \mathrm{COX}-2,{ }^{15}$ vascular endothelial growth factor (VEGF), glyceraldehyde-3-phosphate dehydrogenase (GAPDH) and 28S ribosomal RNA (American Type Culture Collection, Manassas, VA, USA) were used for preparation of radio-labeled probes. Densitometric analysis was performed using Scion Image (Scion Corporation, Frederick, MO, USA).

\section{Assays for Apoptosis}

Morphological examination was performed by phase-contrast microscopy and fluorescence microscopy. For the latter, cells were fixed in $2 \%$ formaldehyde for $10 \mathrm{~min}$ and stained with Hoechst33258 $(10 \mu \mathrm{g} / \mathrm{ml})$ for an additional $30 \mathrm{~min}$. Apoptosis was identified using morphological criteria including shrinkage of the cytoplasm (round shape) and nuclear condensation. Assays were performed in quadruplicate. TUNEL assay was also performed to identify apoptotic cells in the bladder using Apoptosis Detection System, Fluorescein (Promega, Madison, WI, USA), as described previously. ${ }^{16}$ Nuclei were stained with DAPI.

\section{Immunocytochemistry and Immunohistochemistry}

For immunochemistry, cells and tissue sections (frozen sections) on glass slides were fixed in $4 \%$ formaldehyde for $10 \mathrm{~min}$ and permeabilized in $0.2 \%$ Triton-X-100 for $5 \mathrm{~min}$. Staining of $\alpha$-actin was performed using an anti- $\alpha$ smooth muscle actin antibody (Sigma-Aldrich Japan).

\section{Statistical Analysis}

Data were expressed as means \pm s.e. Statistical analysis was performed using the non-parametric Mann-Whitney $U$-test to compare data in different groups. $P$-value $<0.05$ was considered to indicate a statistically significant difference.

\section{RESULTS}

Induction of Mechanical Stress, Hypoxic Stress and ER Stress in the Bladder by Outlet Obstruction

In BOO, mechanical strain and ischemia/hypoxia have been regarded as triggers that cause structural and functional alterations in the bladder. Because mechanical stress and hypoxia have the potential to induce UPR, ER stress caused by these factors may contribute to the damage of the bladder in BOO. To test this hypothesis, we first examined induction of mechanical stress, hypoxic stress and ER stress in the bladder subjected to BOO. An experimental model of BOO was established as described in Materials and Methods, and after $24 \mathrm{~h}$, expression of endogenous indicators for individual stress was evaluated by northern blot analysis. The indicators used were $C O X-2$ for mechanical stress, ${ }^{17}$ GAPDH and VEGF for hypoxic stress ${ }^{18-20}$ and GRP78 and CHOP for ER stress. ${ }^{7,21}$ Northern blot analysis showed that, compared with untreated and sham-operated rats, rats subjected to $\mathrm{BOO}$ exhibited induction of COX-2 (Figure 1a), GAPDH and VEGF (Figure 1b) in the bladders. The induction of mechanical and hypoxic stress was in parallel with induction of

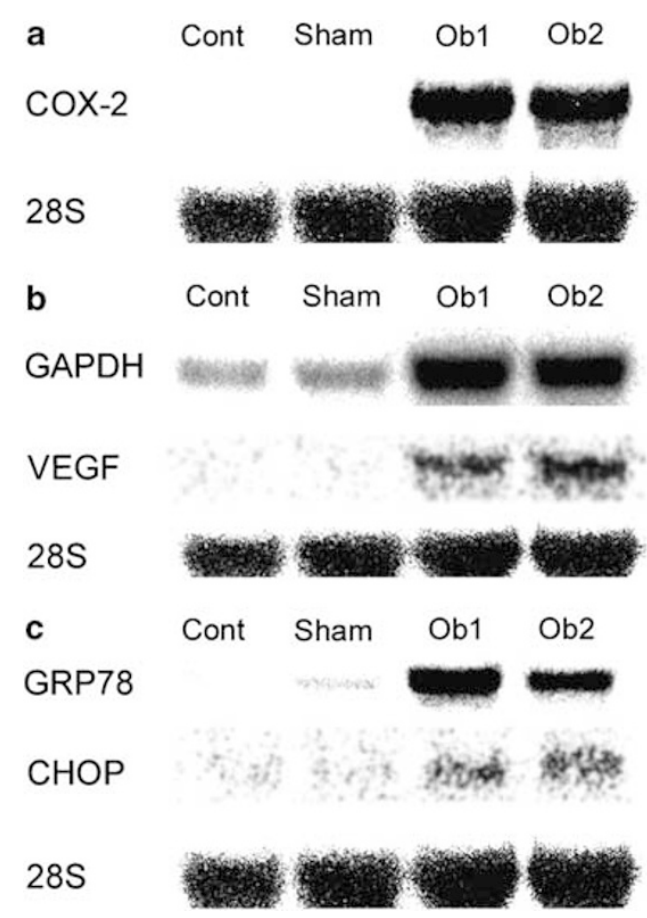

Figure 1 Induction of mechanical stress, hypoxic stress and ER stress in the bladder by outlet obstruction. BOO was established in rats by ligature of the urethra for $24 \mathrm{~h}$. Bladders from untreated control (Cont), sham-operated control (Sham) and BOO-induced rats (Ob1, Ob2) were subjected to northern blot analysis of COX-2, GAPDH, VEGF, GRP78 and CHOP. The level of $28 \mathrm{~S}$ ribosomal RNA is shown as a loading control. The representative results of four independent experiments are shown.

GRP78 and CHOP (Figure 1c). These results suggested that BOO induced mechanical stress and hypoxic stress in the bladder, which was associated with induction of ER stress.

\section{Involvement of Hypoxic Stress, but not Mechanical Stress, in the Induction of ER Stress by BOO}

In the heart, mechanical stress caused by pressure overload induces ER stress that contributes to apoptosis of cardiocytes and heart failure. ${ }^{6}$ To examine whether mechanical stress is causative of the induction of ER stress by BOO, the bladders were distended in vivo by instillation with solution (Figure 2a). Distended bladders were then isolated and incubated ex vivo for $6 \mathrm{~h}$ in an organ bath apparatus, and expression of GRP78 and CHOP as well as COX-2 was evaluated. Northern blot analysis showed that substantial expression of $C O X-2$ was observed, suggesting the induction of mechanical stress (Figure 2b, top row). Under this experimental condition, however, induction of GRP78 and CHOP was not observed (Figure 2b, second and third rows), suggesting that induction of ER stress in BOO was not due to mechanical stress. To further confirm this conclusion, BSMCs were established from bladders, and effects of mechanical stress on the expression of ER stress markers were evaluated using an 
a

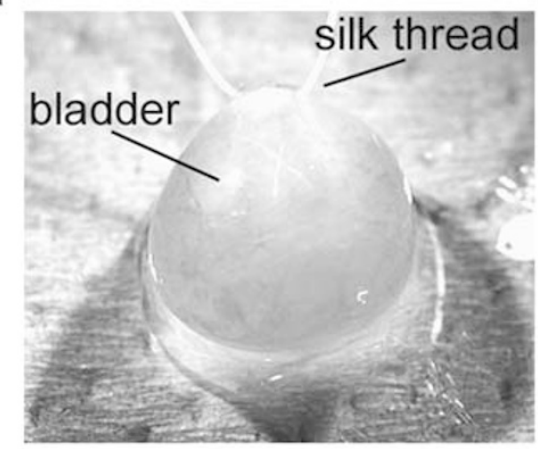

b

coX-2

GRP78

CHOP

$28 S$
Cont Dist

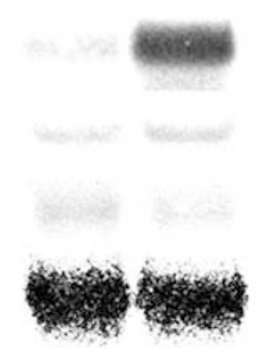

Figure 2 Lack of involvement of mechanical stress in the induction of ER stress in the bladder. $(\mathbf{a}, \mathbf{b})$ Bladders were distended in vivo by instillation with solution, and isolated bladders (a) were incubated ex vivo for $6 \mathrm{~h}$ in an organ bath apparatus. After the incubation, nondistended (Cont) and distended (Dist) bladders were subjected to northern blot analysis of COX-2, GRP78 and CHOP (b). (c) Characterization of primary culture of BSMCs by phase-contrast microscopy (left) and fluorescent microscopy for $\alpha$-actin (right). (d, e) BSMCs were cultured on a deformable silicone chamber (d) and stretched via elongation of the chamber by $40 \%$ for indicated time periods (e). Expression of GRP78 and CHOP was examined by northern blot analysis.

C
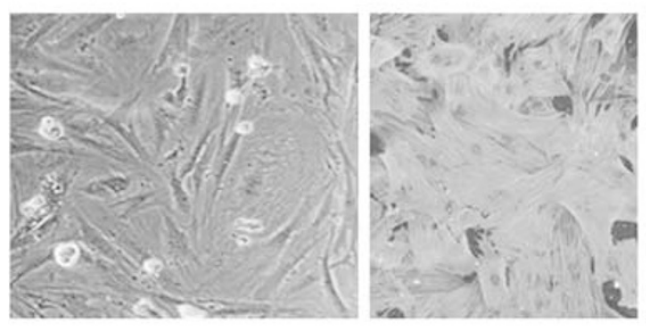

d

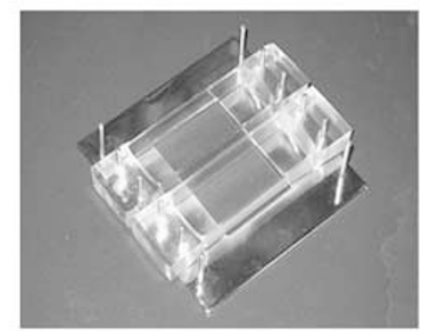

e

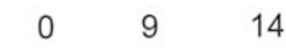

24

(h)

GRP78

CHOP

$28 \mathrm{~S}$

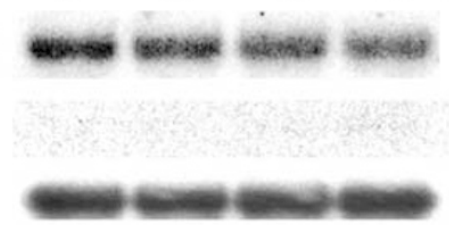

in vitro model of mechanical stress. The established cells showed a spindle shape (Figure 2c, left) and were strongly positive for $\alpha$-actin (Figure $2 c$, right). BSMCs were then cultured on deformable silicone chambers (Figure 2d) and stretched by $40 \%$ for $9-24 \mathrm{~h}$. Consistent with the result of the ex vivo experiment using isolated bladders, expression of GRP78 and CHOP was not induced in BSMCs (Figure 2e). Of note, stretching by more than $40 \%$ caused detachment of the cells from the silicone chambers.

ER stress is induced by a variety of triggers including hypoxia. $^{7}$ We examined a possible role of hypoxia in the induction of ER stress by BOO. For this purpose, BSMCs were exposed to agents that mimic hypoxia in vitro. TTFA and $\mathrm{CoCl}_{2}$ were used for this purpose. TTFA mimics hypoxia by inhibiting the $\mathrm{Q}$ reduction site of mitochondrial respiratory complex II, ${ }^{22}$ and $\mathrm{CoCl}_{2}$ does so by stabilizing hypoxia-inducible factor- $1 \alpha^{23}$ As shown in Figure 3a, treatment of BSMCs with TTFA or $\mathrm{CoCl}_{2}$ significantly induced $V E G F$, confirming induction of hypoxia. Under this experimental condition, expression of GRP78 and CHOP was also induced by TTFA or $\mathrm{CoCl}_{2}$ dose-dependently (Figure $3 \mathrm{~b}$ ). These results suggested a possibility that induction of ER stress in the bladder was due to hypoxia but not mechanical stress.

\section{Induction of Apoptosis in the Bladder by $\mathrm{BOO}$}

As demonstrated, BOO caused ER stress in the bladder. ER stress is known to induce apoptosis in various cell types through activation of caspase-12, phosphorylation of JNK and/or induction of $\mathrm{CHOP}^{24}$ To examine whether or not outlet obstruction causes apoptosis in the bladder, TUNEL assay was performed. Fluorescence microscopy showed that in control bladders, TUNEL-positive cells were hardly detected (Figure $4 \mathrm{a}$, left). In contrast, in the bladders subjected to BOO, a number of TUNEL-positive apoptotic cells were observed (Figure $4 \mathrm{a}$, right). Double-staining with $\alpha$-actin showed that the TUNEL-positive apoptotic cells were located in both the $\alpha$-actin-positive smooth muscle layer and the $\alpha$-actin-negative epithelial layer (Figure $4 b$ ).

\section{Induction of Apoptosis in BSMCs by Hypoxic Stress}

As shown in Figure 3, hypoxia caused ER stress in BSMCs. We examined whether hypoxia has the potential to induce apoptosis in BSMCs. Cultured BSMCs were exposed to $500 \mu \mathrm{M}$ TTFA or $1000 \mu \mathrm{M} \mathrm{CoCl}_{2}$ for $8 \mathrm{~h}$ and subjected to phase-contrast microscopy and Hoechst staining. As shown in Figure 5a, both TTFA and $\mathrm{CoCl}_{2}$ caused shrinkage of the cytoplasm (top row) and nuclear condensation (middle and 


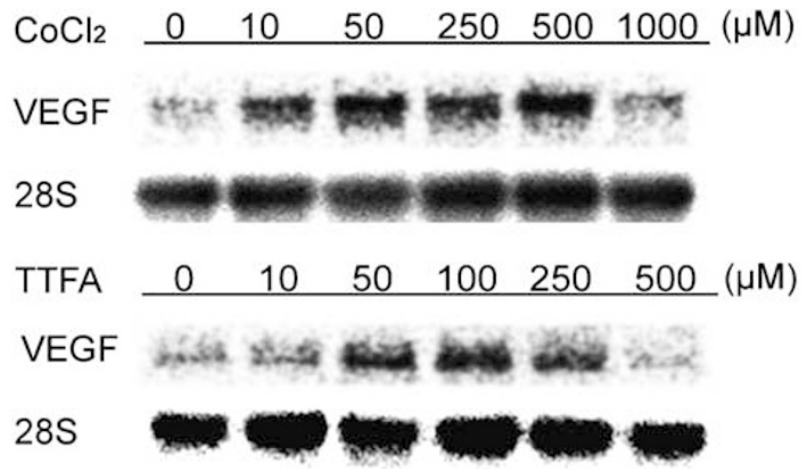

b
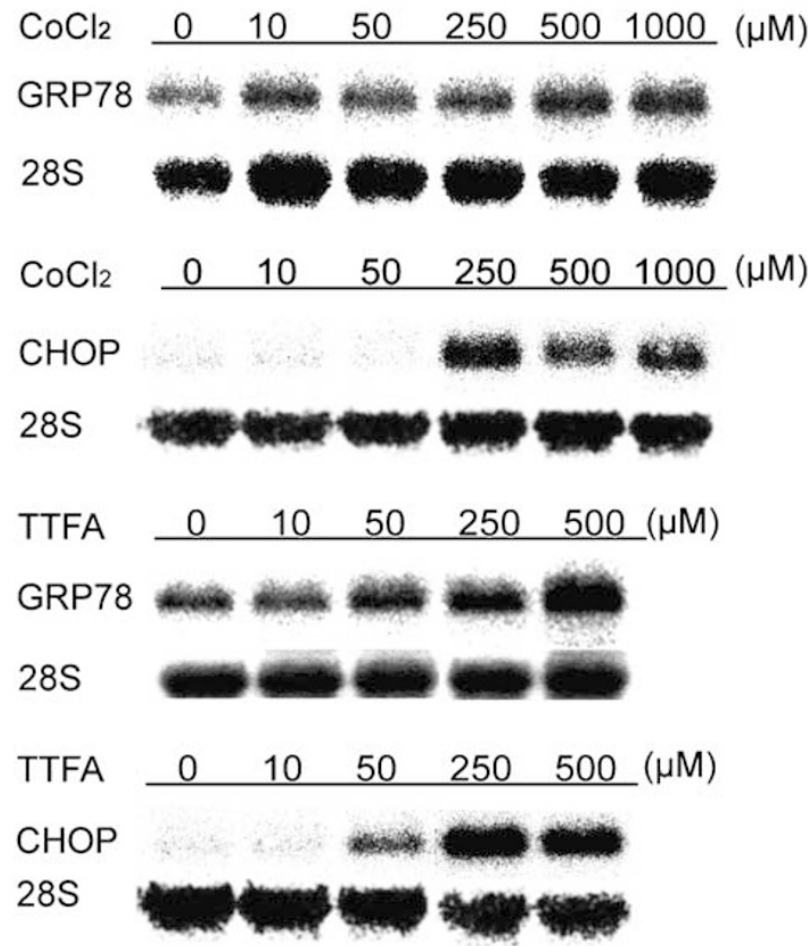

Figure 3 Involvement of hypoxic stress in the induction of ER stress in BSMCs. Cultured BSMCs were exposed to serial concentrations of TTFA or $\mathrm{CoCl}_{2}$ for $8 \mathrm{~h}$, and expression of $\operatorname{VEGF}(\mathbf{a})$, GRP78 and CHOP (b) was examined by northern blot analysis.

bottom rows) typical of apoptosis. To examine the magnitude-dependent effects of hypoxic stress, BSMCs were exposed to TTFA or $\mathrm{CoCl}_{2}$ at serial concentrations and subjected to Hoechst staining to evaluate percentages of apoptotic cells. Significant induction of apoptosis was observed by TTFA at concentrations $\geq 50 \mu \mathrm{M}$ (Figure $5 \mathrm{~b}$ ) and by $\mathrm{CoCl}_{2}$ at concentrations $\geq 250 \mu \mathrm{M}$ (Figure $5 \mathrm{c}$ ). Apoptosis over $80 \%$ was achieved at $500 \mu \mathrm{M}$ of TTFA and $1000 \mu \mathrm{M}$ of $\mathrm{CoCl}_{2}$, respectively.

Attenuation of Hypoxia-Induced Apoptosis In vitro and BOO-Induced Apoptosis In vivo by Chemical Chaperone In vitro, hypoxic stress caused apoptosis of BSMCs. To investigate whether ER stress is involved in the induction of apoptosis caused by hypoxic stress, a chemical chaperone was used to attenuate ER stress. A previous report showed that 4-PBA facilitates normal trafficking of mutant proteins from the ER to the plasma membrane. ${ }^{25}$ Other reports also showed that 4-PBA has chemical chaperone activity and protects the brain against ischemic injury and ER stress-induced neuronal death in vivo and in vitro. ${ }^{26,27}$ Using 4-PBA, we first examined whether ER stress triggered by tunicamycin and thapsigargin, known inducers of ER stress, is attenuated by 4-PBA in BSMCs. BSMCs were pretreated with 4-PBA and stimulated with tunicamycin or thapsigargin for $8 \mathrm{~h}$. Northern blot analysis revealed that 4-PBA reduced induction of GRP78 and CHOP by the ER stress inducers (Figure 6a, left). Consistent with this result, Hoechst staining revealed that 4-PBA significantly suppressed apoptotic cell death induced by tunicamycin (Figure 6a, right). On the basis of these results, we next examined whether or not hypoxia-induced ER stress and apoptosis can be attenuated by 4-PBA. As shown in Figure 6b, treatment with TTFA induced expression of GRP78 and CHOP, and pretreatment of the cells with 4-PBA suppressed this induction. Furthermore, microscopic analysis revealed that cellular damage induced by TTFA or $\mathrm{CoCl}_{2}$ was attenuated by treatment with 4-PBA (Figure 6c). Quantitative analysis using Hoechst staining showed that both TTFA- and $\mathrm{CoCl}_{2}$-induced apoptosis was significantly decreased by treatment with 4-PBA (Figure 6d). The decreases in percentages of apoptotic cells were from $33.3 \pm 2.6$ to $21.6 \pm 6.0 \%$ in TTFA-treated cells and from $30.0 \pm 5.9$ to $15.4 \pm 1.3 \%$ in $\mathrm{CoCl}_{2}$-treated cells (means \pm s.e., $P<0.05$ ). These results suggested the involvement of ER stress in hypoxia-induced apoptosis of BSMCs.

To investigate whether ER stress triggered by hypoxia is also involved in the induction of apoptosis by BOO, the in vivo effects of 4-PBA were tested. Rats were administered intraperitoneally with 4-PBA for 3 days, and bladders were subjected to $\mathrm{BOO}$ for $24 \mathrm{~h}$. TUNEL assay showed that induction of apoptosis by BOO was attenuated by 4-PBA (Figure 7a). The percentage of apoptotic cells in the $\mathrm{BOO}$ group without 4 -PBA was $5.5 \pm 1.1 \%$ per high-power field, and treatment with 4-PBA significantly reduced the apoptosis to $1.4 \pm 0.7 \%$ per field (means \pm s.e., $P<0.05$ ) (Figure $7 \mathrm{~b}$ ). This result suggested that ER stress triggered by hypoxia may be involved in the induction of apoptosis in the bladder suffered from BOO.

\section{DISCUSSION}

In the present study, we provide the first evidence for induction of ER stress in the bladder subjected to BOO. We suggest a possibility that ER stress is caused not via mechanical stress but through hypoxic stress and that hypoxia-induced ER stress is causative of apoptotic cell death in the bladder following outlet obstruction.

The presence of hypoxia in the models of BOO has been documented by several investigators. For example, Levin et $\mathrm{al}^{28}$ reported that, in a rabbit model of BOO, focal hypoxia 
a
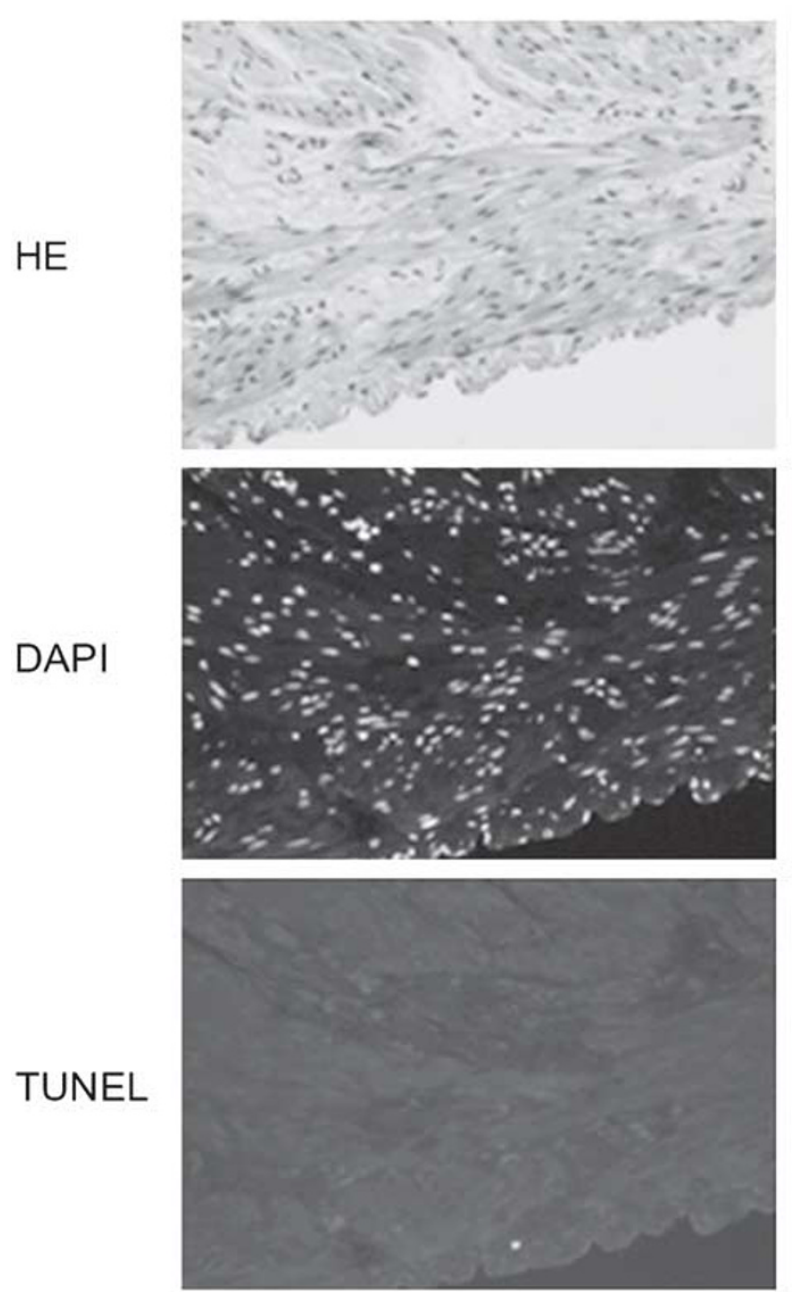

b

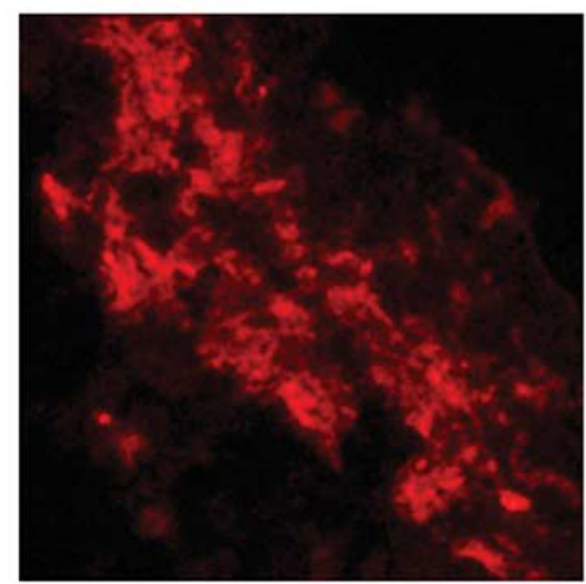

a-actin
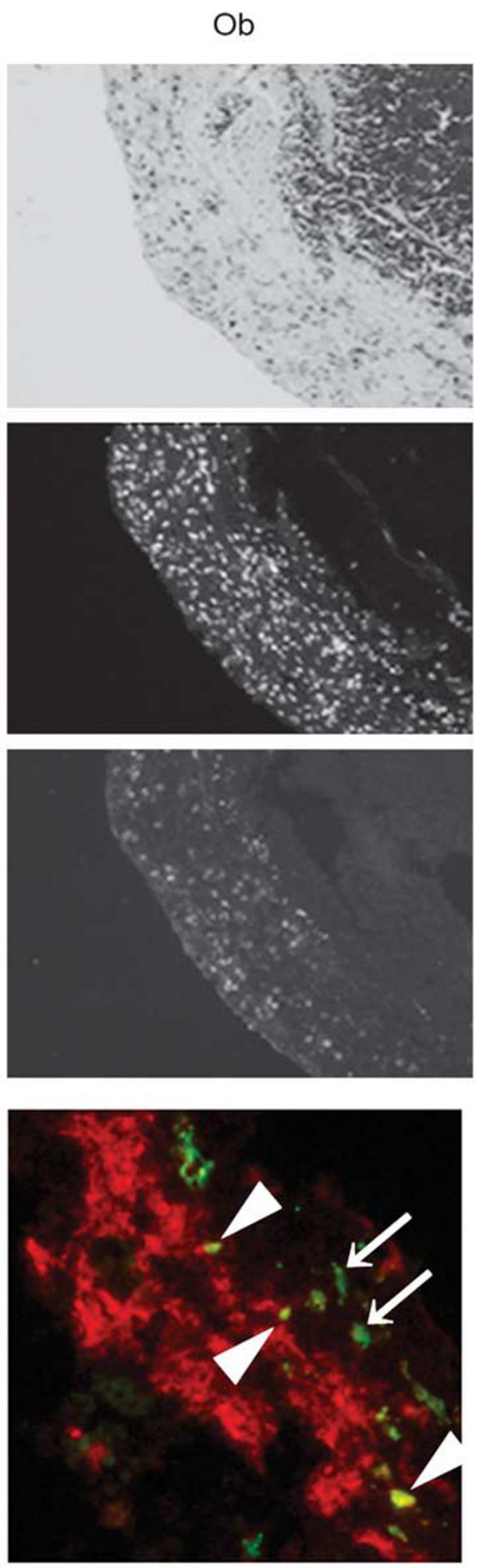

TUNEL+a-actin

Figure 4 Induction of apoptosis in the bladder by BOO. (a) Control bladders (Cont) and bladders subjected to BOO for $24 \mathrm{~h}(\mathrm{Ob})$ were examined histologically by hematoxylin-eosin staining (HE), DAPI staining and TUNEL. (b) Bladders from BOO rats were double-stained with $\alpha$-actin (red) and TUNEL (green) and subjected to fluorescent microscopy. Apoptotic non-BSMCs (green) and apoptotic BSMCs (yellow) were indicated by arrows and arrowheads, respectively. 
a

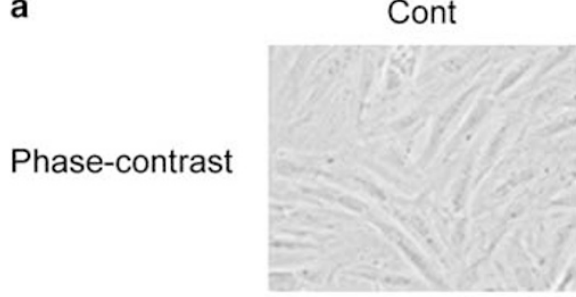

Hoechst

Low magnification
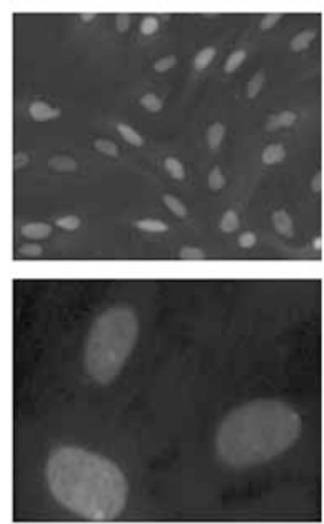

Hoechst

High magnification
TTFA
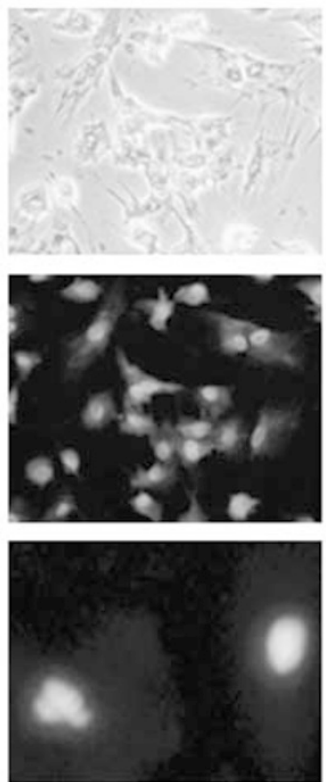

$\mathrm{CoCl}_{2}$
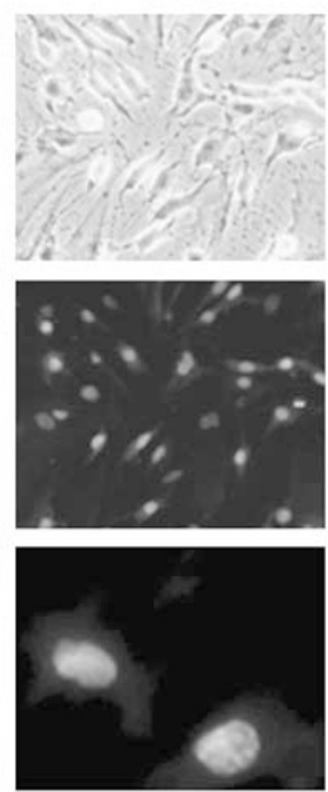
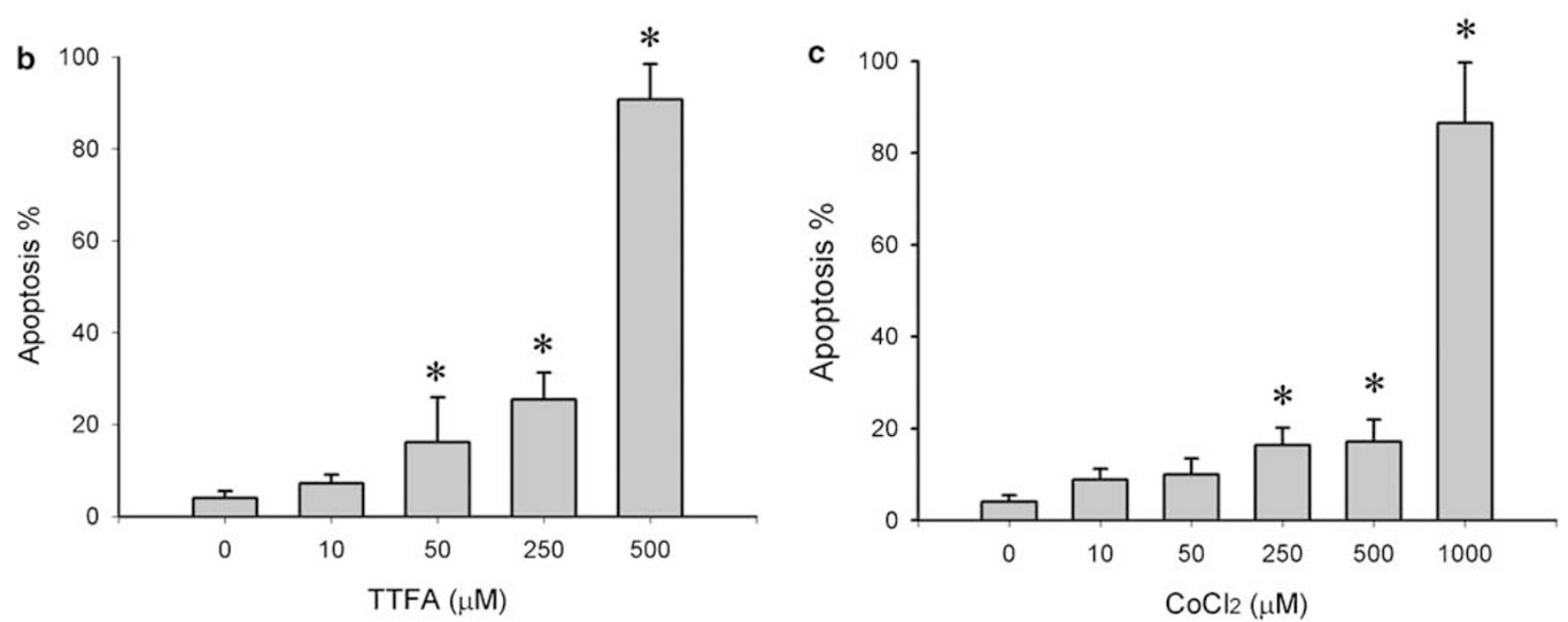

Figure 5 Induction of apoptosis in cultured BSMCs by hypoxia. (a) BSMCs were exposed to $500 \mu \mathrm{M}$ TTFA or $1000 \mu \mathrm{M} \mathrm{CoCl}$ for $8 \mathrm{~h}$ and subjected to phasecontrast microscopy (top) and Hoechst staining (middle, low magnification; bottom, high magnification). (b, c) After exposure to serial concentrations of TTFA (b) or $\mathrm{CoCl}_{2}$ (c), percentages of apoptotic cells with condensed nuclei were evaluated by fluorescent microscopy. Assays were performed in quadruplicate, and data are expressed as means \pm s.e. ${ }^{*}$ Significantly different vs untreated control $(P<0.05)$.

was detected in the detrusor smooth muscles and subserosal regions of the bladders. They also showed that it was associated with decreased contractile function and selective metabolic dysfunction of smooth muscles. Of note, hypoxia was not observed in the bladder mucosa. ${ }^{28}$ Azadzoi et al reported (1) bladder filling caused a significant decrease in blood flow and oxygen tension even without outlet obstruction and (2) spontaneous bladder contraction resulted in a decrease in bladder wall perfusion in the obstructed bladder. ${ }^{3}$ In consistence with these previous reports, our current results showed upregulation of hypoxic stress markers, GAPDH and $V E G F$, in the bladder subjected to BOO.
Hypoxia is known to induce ER stress in various cells and tissues and thereby regulates cell survival. ${ }^{7}$ For example, ischemic/hypoxic insults in the brain and heart caused ER stress-dependent apoptosis of resident cells and thereby induced injury and dysfunction of these organs. ${ }^{8}$ However, molecular mechanisms involved in the hypoxia-triggered ER stress have not been fully elucidated. In yeasts, dedicated machinery of molecular chaperones and foldases that associate directly with nascent polypeptides assists correct folding of proteins. Foldases catalyze formation of disulfide linkages by transiently forming mixed disulfides with their client proteins and acting as an electron relay system for oxidative 


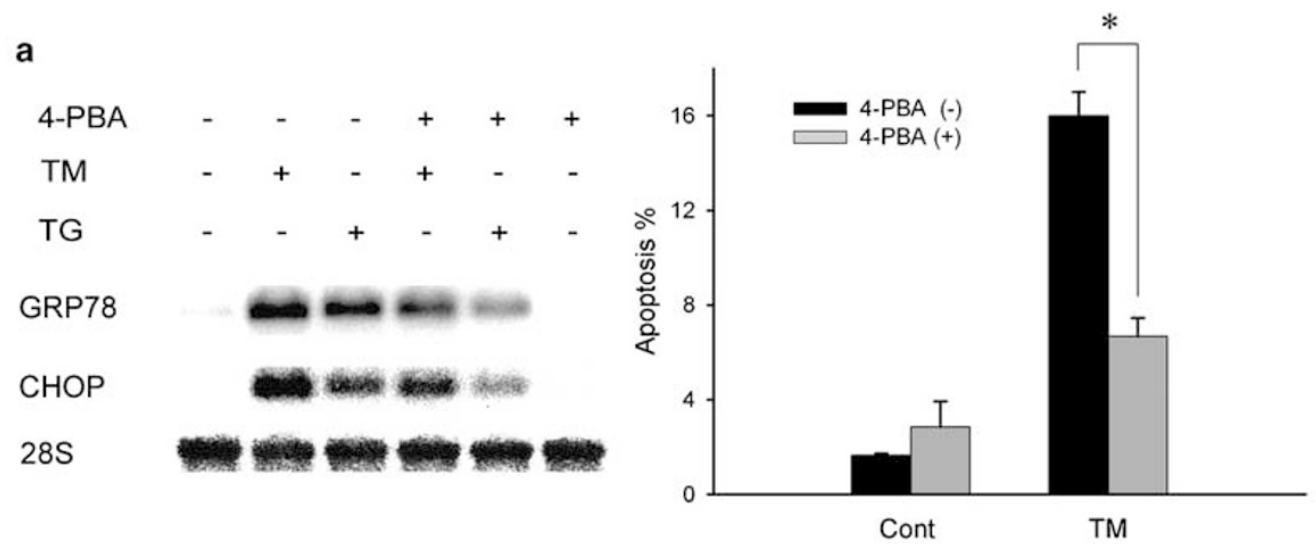

b

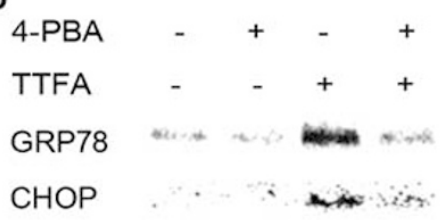

$28 S$ Has

c

Cont
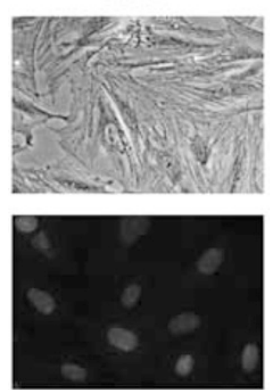

4-PBA
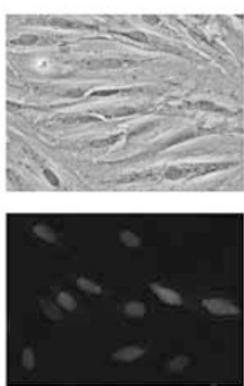
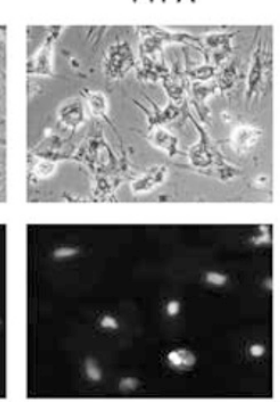

$\underset{+}{4-P B A}$

TTFA
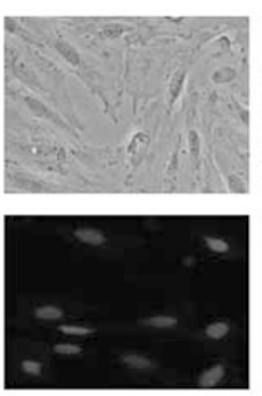

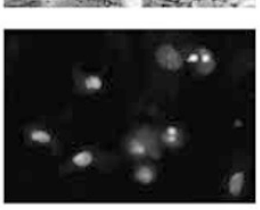

$\mathrm{CoCl}_{2}$
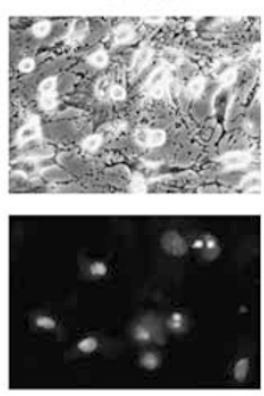
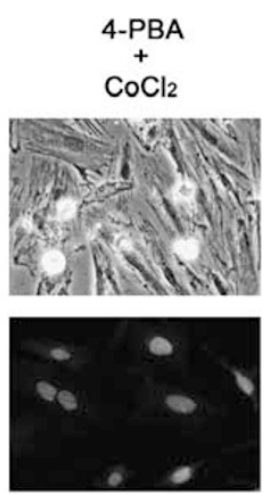

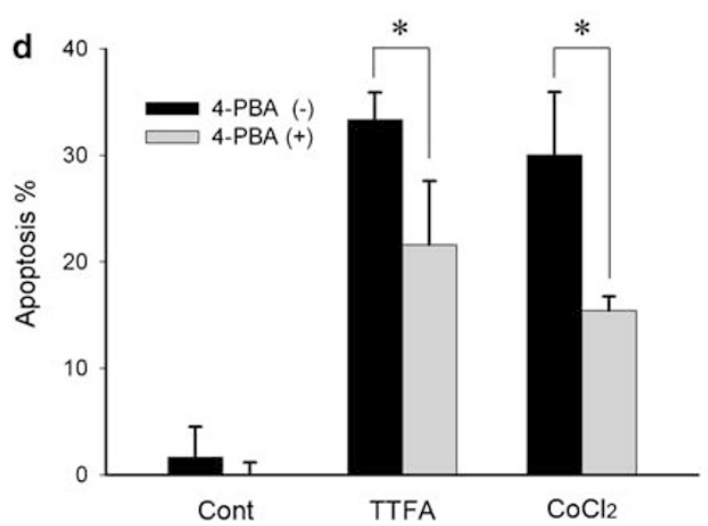

Figure 6 Attenuation of hypoxia-induced apoptosis of BSMCs by chemical chaperone. (a, b) BSMCs were pretreated with (+) or without (-) 4-PBA (5 mM) and exposed to tunicamycin (TM; $5 \mu \mathrm{g} / \mathrm{ml}$ ) or thapsigargin (TG; $100 \mathrm{nM})(\mathbf{a})$, or TTFA $(500 \mu \mathrm{M})(\mathbf{b})$ for $8 \mathrm{~h}$. Expression of GRP78 and CHOP was examined by northern blot analysis. In (a), quantitative analysis of TM-induced apoptosis in the absence (-) or presence (+) of 4-PBA is shown on the right. (c, d) BSMCs were pretreated with or without 4-PBA, exposed to TTFA or $\mathrm{CoCl}_{2}$ for $8 \mathrm{~h}$ and subjected to phase-contrast microscopy (c, top) and Hoechst staining (c, bottom). Percentages of apoptotic cells with condensed nuclei were evaluated by fluorescent microscopy (d). Data are expressed as means \pm s.e. Asterisks indicate statistically significant differences $(P<0.05)$. 
a

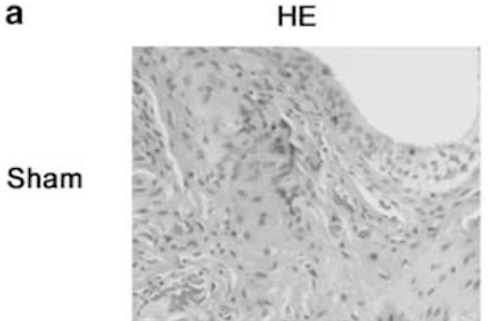

4-PBA

$+$

Sham

$\mathrm{Ob}$
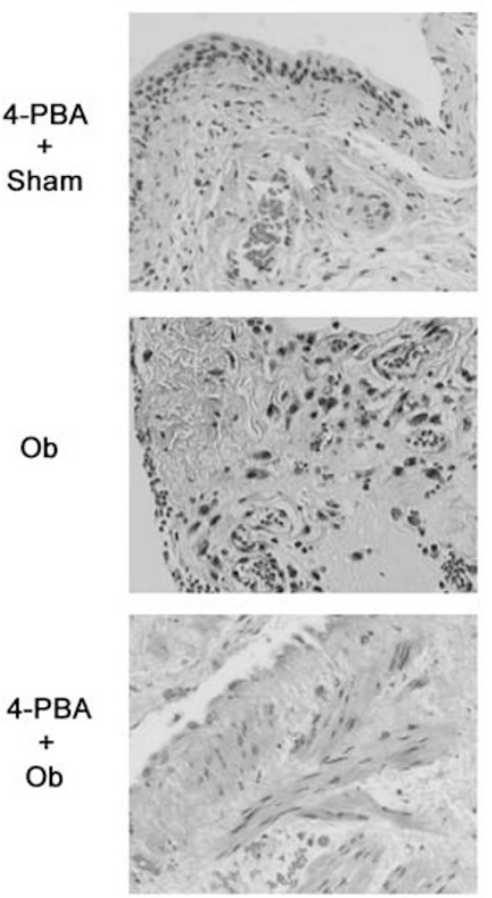

DAPI
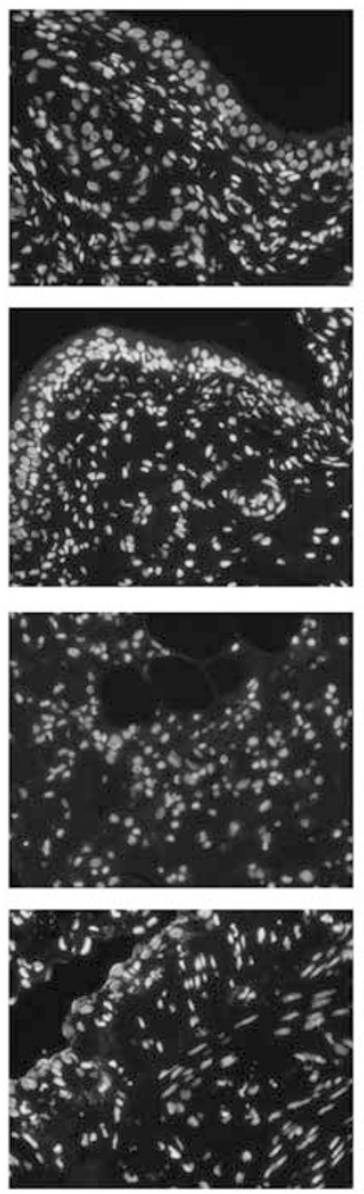

TUNEL
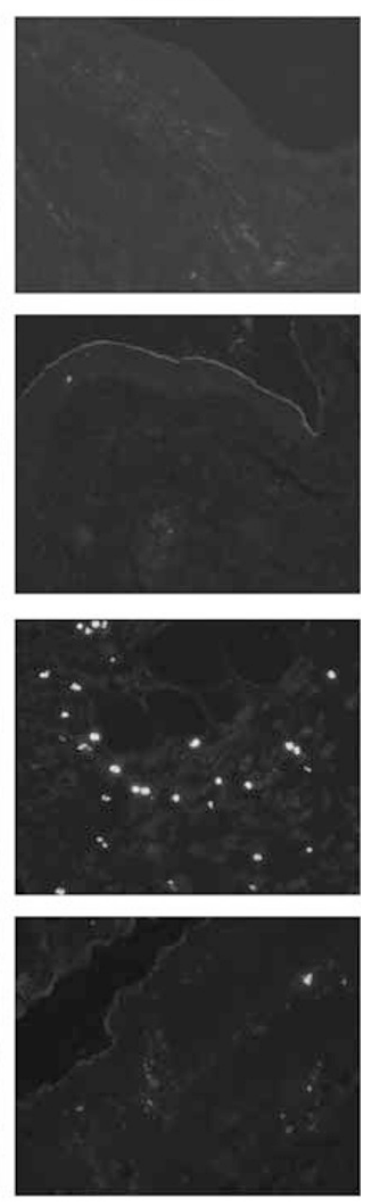

Figure 7 Attenuation of BOO-induced apoptosis in the bladder by chemical chaperone. Rats were administered daily with either ethanol or 4-PBA ( $120 \mathrm{mg} / \mathrm{kg}$, i.p.) for 3 days and subjected to BOO. Histological analyses were performed using HE staining, DAPI staining and TUNEL assay. Assays were repeated four times, and representative data are shown in (a). Percentages of TUNEL-positive cells were evaluated quantitatively and shown in (b). Data are expressed as means \pm s.e. An asterisk indicates a statistically significant difference $(P<0.05)$.

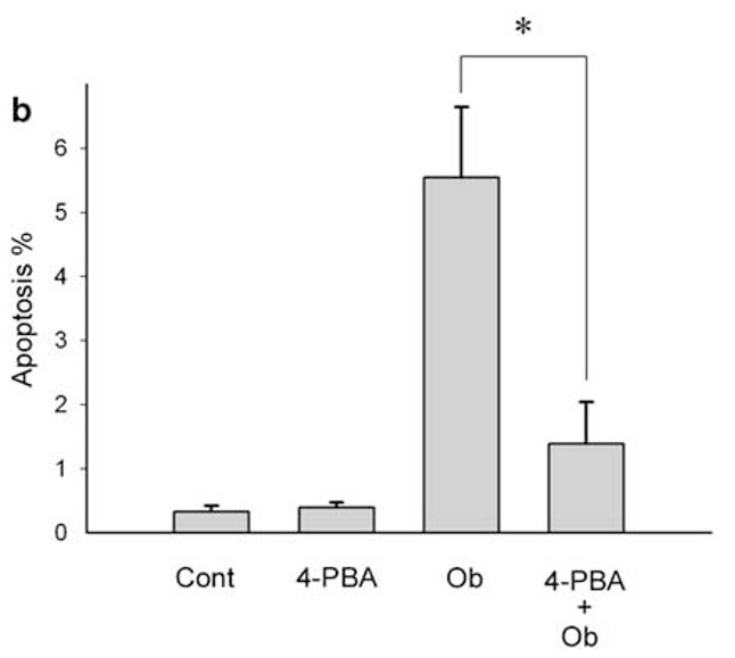

folding. ${ }^{29}$ Molecular oxygen is the major electron acceptor at the end of this relay system, providing the driving force for protein folding in the ER. In mammalian cells, this pathway may be similarly dependent on molecular oxygen, and folding of proteins in the ER may be disturbed in the absence of oxygen (hypoxia), ${ }^{30}$ leading to an accumulation of unfolded proteins in the ER.

In this report, we showed that hypoxia-triggered ER stress was involved in the induction of apoptosis by BOO. Under ER stress conditions, several death signals may be transduced 
and contribute to apoptosis. For example, $\mathrm{CHOP}$, also called growth arrest and DNA damage-inducible protein 153, is upregulated via transcription factor 4 , which is induced by the RNA-dependent protein kinase-like ER kinase-eukaryotic translation initiation factor $2 \alpha$ pathway. ${ }^{24}$ We found that $\mathrm{CHOP}$ was induced in the bladders subjected to BOO, indicating a possible contribution of this molecule to BOOtriggered, hypoxia- and ER stress-mediated apoptosis. ER stress also activates ER stress-specific caspase-12 localized at the ER membrane through an interaction with inositolrequiring ER-to-nucleus signal kinase 1 (IRE1) and tumor necrosis factor receptor-associated factor 2 (TRAF2). In humans, the caspase- 12 gene is a pseudogene, and caspase- 4 functions as an ER stress-specific caspase. The IRE1-TRAF2 interaction also allows for recruitment and activation of apoptosis signal-regulating kinase 1 and downstream JNK, both of which are involved in a variety of apoptotic pathways. ${ }^{24}$ Further investigation will be required to identify the exact branches of UPR and downstream molecules responsible for the induction of apoptosis.

4-PBA is a low molecular weight compound that stabilizes protein conformation, improves folding capacity of the ER and facilitates trafficking of mutant proteins. ${ }^{31} 4$-PBA has been demonstrated to reduce the load of mutant or mislocated proteins retained in the ER under conditions associated with cystic fibrosis and liver injury. ${ }^{25}$ It is also reported that 4-PBA has a neuroprotective effect on cerebral ischemic injury in a mouse model of ischemia/hypoxia. ${ }^{27}$ In the present study, we showed that 4-PBA attenuated hypoxiainduced apoptosis in BSMCs. Furthermore, we also demonstrated that systemic administration with 4-PBA at a therapeutic dosage reduced the number of apoptotic cells in outlet-obstructed bladder. This result suggested an in vivo role of ER stress in the induction of apoptosis by BOO. However, the mechanisms by which 4-PBA elicits proper maturation of unfolded proteins have not been fully elucidated, and further investigation may be required to confirm our conclusion.

The chemical chaperone 4-PBA is a safe agent that has already been approved by the US Food and Drug Administration for the treatment of urea cycle disorders in children, thalassemia and cystic fibrosis. ${ }^{32}$ ER stress-induced apoptosis plays crucial roles in the pathogenesis of a number of human diseases, including hypoxia and ischemia, viral infection, neurodegenerative disorders, drug-induced tissue injury, and metabolic diseases such as diabetes mellitus. ${ }^{7}$ Our present data, together with previous reports, showed that apoptosis is induced in both urothelial cells and smooth muscle cells and may contribute to hypocellularity and fibrosis in the outlet-obstructed bladders. ${ }^{33,34}$ Although currently pathological significance of apoptosis in the bladder during the course of $\mathrm{BOO}$ is not determined, our present findings indicated a possibility that chemical chaperones have the potential to attenuate functional and/or histopathological abnormalities observed in the bladders suffered from outlet obstruction.

\section{ACKNOWLEDGEMENT}

This work was supported, in part, by Grant-in-Aids for Scientific Research from the Ministry of Education, Culture, Sports, Science, and Technology of Japan (Nos. 16390243, 17651026 and 19651024 to M K and No. 19591840 to I A).

1. Steers WD, De Groat WC. Effect of bladder outlet obstruction on micturition reflex pathways in the rat. J Urol 1988;140:864-871.

2. Levin RM, Wein AJ, Buttyan R, et al. Update on bladder smooth-muscle physiology. World J Urol 1994;12:226-232.

3. Azadzoi KM, Pontari M, Vlachiotis J, et al. Canine bladder blood flow and oxygenation: changes induced by filling, contraction and outlet obstruction. J Urol 1996;155:1459-1465.

4. Yamaguchi $O$. Response of bladder smooth muscle cells to obstruction: signal transduction and the role of mechanosensors. Urology 2004;63:11-16.

5. Ron D. Translational control in the endoplasmic reticulum stress response. J Clin Invest 2002;110:1383-1388.

6. Okada K, Minamino T, Tsukamoto $\mathrm{Y}$, et al. Prolonged endoplasmic reticulum stress in hypertrophic and failing heart after aortic constriction: possible contribution of endoplasmic reticulum stress to cardiac myocyte apoptosis. Circulation 2004;110:705-712.

7. Xu C, Bailly-Maitre B, Reed JC. Endoplasmic reticulum stress: cell life and death decisions. J Clin Invest 2005;115:2656-2664.

8. Thuerauf DJ, Marcinko M, Gude N, et al. Activation of the unfolded protein response in infarcted mouse heart and hypoxic cultured cardiac myocytes. Circ Res 2006;99:275-282.

9. $\mathrm{Ma} F \mathrm{H}$, Higashira-Hoshi $\mathrm{H}$, Itoh $\mathrm{Y}$. Functional muscarinic $\mathrm{M}_{2}$ and $\mathrm{M}_{3}$ receptors and $\beta$-adrenoceptor in cultured rat bladder smooth muscle. Life Sci 2002;70:1159-1172.

10. Hsu SM, Raine L, Fanger H. Use of avidin-biotin-peroxidase complex $(A B C)$ in immunoperoxidase techniques: a comparison between $A B C$ and unlabeled antibody (PAP) procedures. J Histochem Cytochem 1981;29:577-580.

11. Komuro I, Kaida T, Shibazaki $Y$, et al. Stretching cardiac myocytes stimulates protooncogene expression. J Biol Chem 1990;265: 3595-3598.

12. Kitamura $M$, Sütö $T$, Yokoo $T$, et al. Transforming growth factor- $\beta 1$ is the predominant paracrine inhibitor of macrophage cytokine synthesis produced by glomerular mesangial cells. J Immunol 1996;156:2964-2971.

13. Katayama T, Imaizumi K, Honda A, et al. Disturbed activation of endoplasmic reticulum stress transducers by familial Alzheimer's disease-linked presenilin-1 mutations. J Biol Chem 2001;276: 43446-43454.

14. Wang $X Z$, Harding $H P$, Zhang $Y$, et al. Cloning of mammalian Ire1 reveals diversity in the ER stress responses. EMBO $J$ 1998;17:5708-5717.

15. DeWitt $\mathrm{DL}$, Meade EA. Serum and glucocorticoid regulation of gene transcription and expression of the prostaglandin $\mathrm{H}$ synthase- 1 and prostaglandin $\mathrm{H}$ synthase-2 isozymes. Arch Biochem Biophys 1993;306:94-102.

16. Yokouchi M, Hiramatsu N, Hayakawa K, et al. Atypical, bidirectional regulation of cadmium-induced apoptosis via distinct signaling of unfolded protein response. Cell Death Differ 2007;14:1467-1474.

17. Park JM, Yang T, Arend LJ, et al. Obstruction stimulates COX-2 expression in bladder smooth muscle cells via increased mechanical stretch. Am J Physiol 1999;276:F129-F136.

18. Graven KK, Troxler RF, Kornfeld $\mathrm{H}$, et al. Regulation of endothelial cell glyceraldehyde-3-phosphate dehydrogenase expression by hypoxia. J Biol Chem 1994;269:24446-24453.

19. Lu S, Gu X, Hoestje $S$, et al. Identification of an additional hypoxia responsive element in the glyceraldehyde-3-phosphate dehydrogenase gene promoter. Biochim Biophys Acta 2002;1574: 152-156.

20. Burgu B, Medina Ortiz WE, Pitera JE, et al. Vascular endothelial growth factor mediates hypoxic stimulated embryonic bladder growth in organ culture. J Urol 2007;177:1552-1557.

21. Lee AS. The accumulation of three specific proteins related to glucoseregulated proteins in a temperature-sensitive hamster mutant cell line K12. J Cell Physiol 1981;106:119-125. 
22. Sun $F$, Huo $X$, Zhai $Y$, et al. Crystal structure of mitochondrial respiratory membrane protein complex II. Cell 2005;121:1043-1057.

23. Corley KM, Taylor CJ, Lilly B. Hypoxia-inducible factor $1 \alpha$ modulates adhesion, migration, and FAK phosphorylation in vascular smooth muscle cells. J Cell Biochem 2005;96:971-985.

24. Kim R, Emi M, Tanabe $K$, et al. Role of the unfolded protein response in cell death. Apoptosis 2006;11:5-13.

25. Zeitlin PL, Diener-West M, Rubenstein RC, et al. Evidence of CFTR function in cystic fibrosis after systemic administration of 4-phenylbutyrate. Mol Ther 2002;6:119-126.

26. Kubota K, Niinuma $Y$, Kaneko $M$, et al. Suppressive effects of 4-phenylbutyrate on the aggregation of Pael receptors and endoplasmic reticulum stress. J Neurochem 2006;97:1259-1268.

27. Qi X, Hosoi T, Okuma $Y$, et al. Sodium 4-phenylbutyrate protects against cerebral ischemic injury. Mol Pharmacol 2004;66:899-908.

28. Levin RM, O'Connor LJ, Leggett RE, et al. Focal hypoxia of the obstructed rabbit bladder wall correlates with intermediate decompensation. Neurourol Urodyn 2003;22:156-163.
29. Tu BP, Weissman JS. Oxidative protein folding in eukaryotes: mechanisms and consequences. J Cell Biol 2004;164:341-346.

30. Feldman $D E$, Chauhan $V$, Koong $A C$. The unfolded protein response: a novel component of the hypoxic stress response in tumors. Mol Cancer Res 2005;3:597-605.

31. Vilatoba $M$, Eckstein C, Bilbao $G$, et al. Sodium 4-phenylbutyrate protects against liver ischemia reperfusion injury by inhibition of endoplasmic reticulum-stress mediated apoptosis. Surgery 2005;138:342-351.

32. Carducci MA, Gilbert J, Bowling MK, et al. A phase I clinical and pharmacological evaluation of sodium phenylbutyrate on an $120-\mathrm{h}$ infusion schedule. Clin Cancer Res 2001;7:3047-3055.

33. Chertin B, Rolle U, Solari $V$, et al. The role of nitric oxide in bladder urothelial injury after bladder outlet obstruction. BJU Int 2004;94:392-399.

34. Thiruchelvam N, Nyirady $\mathrm{P}$, Peebles DM, et al. Urinary outflow obstruction increases apoptosis and deregulates $\mathrm{BCl}-2$ and $\mathrm{Bax}$ expression in the fetal ovine bladder. Am J Pathol 2003;162:1271-1282. 